\title{
On the Hilbert function of lines union one non-reduced point
}

\author{
ENRICO CARLINI, MARIA VIRGINIA CATALiSANO \\ AND ANTHONY V. GERAMITA
}

\begin{abstract}
In this paper we consider the problem of determining the Hilbert function of schemes $X \subset \mathbb{P}^{n}$ which are the generic union of $s$ lines and one $m$ multiple point. We completely solve this problem for any $s$ and $m$ when $n \geq 4$. When $n=3$ we find several defective such schemes and conjecture that they are the only ones. We verify this conjecture in several cases.
\end{abstract}

Mathematics Subject Classification (2010): 14N20 (primary); 14C20, 13H15 (secondary).

\section{Introduction}

If $P$ is a point in $\mathbb{P}^{n}$ with corresponding ideal $I_{P} \subset R=k\left[x_{0}, \ldots, x_{n}\right]$, where $k$ is algebraically closed of characteristic zero, the scheme supported on $P$ and defined by the ideal $\left(I_{P}\right)^{m}$ is called an m-multiple point with support $P$. In a remarkable paper [1] J. Alexander and A. Hirschowitz found the Hilbert function of a finite union of 2-multiple points supported on a generic set of points in $\mathbb{P}^{n}$ (see also [8] and [2] for simpler proofs). This result permitted Alexander and Hirschowitz to solve the long open problem regarding the dimensions of the (higher) secant varieties of the Veronese varieties (see $[11,15]$ for an expository discussion of this important result). In a subsequent paper [7] Catalisano, Geramita and Gimigliano showed that, in an analogous way (using the Lemma of Terracini) one can find the dimensions of the (higher) secant varieties to Segre embeddings of products of projective spaces, if one could calculate the Hilbert functions of certain unions of reduced and non-reduced schemes supported on unions of generic linear spaces of different dimensions (for more details see Theorem 1.1 in [7]). The study of such schemes is one of the principal motivations for our work in this paper.

There is also other closely related research in the literature, e.g., some authors have considered the problem of finding the Hilbert function of generic $m$-multiple

All the authors enjoyed support from NSERC (Canada). The first and second authors were also supported by GNSAGA of INDAM and by MIUR (Italy).

Received September 3, 2013; accepted January 20, 2014.

Published online February 2016. 
points in $\mathbb{P}^{2}$ (see the survey [17] and $[6,14,18]$ ) as well as of generic $m$-multiple points in $\mathbb{P}^{n}$ with $n>2$ (see $\left.[9,16]\right)$. Moreover, Hartshorne and Hirschowitz considered the same problem for a generic union of (reduced) lines in $\mathbb{P}^{n}$, with $n>2$. Recent results in this direction, and in the more general setting of Nagata's conjecture, are contained in [10] and [12].

In this paper we consider yet another variant of this family of problems: namely the case in which the scheme $X \subset \mathbb{P}^{n}$, with $n \geq 3$, is composed of $s$ generic (reduced) lines and one generic $m$-multiple point. A simple parameter count leads one to expect that the Hilbert function of such an $X$, denoted $\operatorname{HF}(X, \cdot)$, is

$$
\mathrm{HF}(X, d)=\min \left\{\left(\begin{array}{c}
d+n \\
n
\end{array}\right),\left(\begin{array}{c}
m+n-1 \\
n
\end{array}\right)+s(d+1)\right\} .
$$

If we let $h p(X, \cdot)$ denote the Hilbert polynomial of $X$, then $(*)$ is really saying that

$$
\mathrm{HF}(X, d)=\min \left\{h p\left(\mathbb{P}^{n}, d\right), h p(X, d)\right\},
$$

or equivalently

$$
\operatorname{dim}\left(I_{X}\right)_{d}=\max \left\{\left(\begin{array}{c}
d+n \\
n
\end{array}\right)-\left(\begin{array}{c}
m+n-1 \\
n
\end{array}\right)-s(d+1), 0\right\} .
$$

Note that in this case we say that the Hilbert function of $X$ is bipolynomial (see also [3] for other examples of this).

We prove ( $*$ ) for any $s$ and $m$ when $n \geq 4$ (see Theorem 3.2). When $n=3$, the situation is less clear. In particular, the "simple parameter count" no longer always gives the actual Hilbert function (the precise statement is given in Theorem 4.2). We conjecture that the parameter count fails (for $n=3$ ) if and only if $m=d$ and $1<s \leq d$. In these cases we show that $\operatorname{dim}\left(I_{X}\right)_{d}=\left(\begin{array}{c}d-s+2 \\ 2\end{array}\right)$.

ACKNOWLEDGEMENTS. The first and second authors wish to thank Queens University, in the person of the third author, for their kind hospitality during the preparation of this work.

\section{Basic facts and notation}

Since we will make use of Castelnuovo's inequality several times, we recall it here in a form more suited to our use (for notation and proof we refer to [1, Section 2]).

Definition 2.1. If $X, Y$ are closed subschemes of $\mathbb{P}^{n}$, we denote by $\operatorname{Res}_{Y} X$ the scheme defined by the ideal $\left(I_{X}: I_{Y}\right)$ and we call it the residual scheme of $X$ with respect to $Y$, we denote by $\operatorname{Tr}_{Y} X \subset Y$ the schematic intersection $X \cap Y$, and call it the trace of $X$ on $Y$. We also denote by $X+Y$ the schematic union of $X$ and $Y$. 
Lemma 2.2 (Castelnuovo's inequality). Let $d, \delta \in \mathbb{N}$, with $d \geq \delta$, let $Y \subseteq \mathbb{P}^{n}$ be a smooth hypersurface of degree $\delta$, and let $X \subseteq \mathbb{P}^{n}$ be a closed subscheme. Then

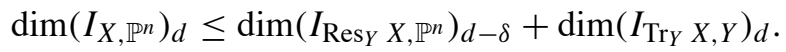

Now an easy, but useful lemma.

Lemma 2.3. Let $X \subset \mathbb{P}^{n}$.

(i) If $X=X_{1}+\cdots+X_{s}$ is the union of non-intersecting closed subschemes $X_{i}$, if $X^{\prime}=X_{1}+\cdots+X_{s^{\prime}} \subset X$, where $s^{\prime}<s$, and if $\operatorname{HF}(X, d)=\sum_{i=1}^{s} \operatorname{HF}\left(X_{i}, d\right)$, then

$$
\operatorname{HF}\left(X^{\prime}, d\right)=\sum_{i=1}^{s^{\prime}} \operatorname{HF}\left(X_{i}, d\right)
$$

(ii) If $X=Y+m P$ is the union of a closed subscheme $Y$ and one m-multiple point, if $X^{\prime}=Y+m^{\prime} P \subset X$, where $m^{\prime}<m$, and if $\operatorname{HF}(X, d)=\operatorname{HF}(Y, d)+\left(\begin{array}{c}m+n-1 \\ n\end{array}\right)$, then

$$
\operatorname{HF}\left(X^{\prime}, d\right)=\operatorname{HF}(Y, d)+\left(\begin{array}{c}
m^{\prime}+n-1 \\
n
\end{array}\right) .
$$

(iii) If $\operatorname{dim}\left(I_{X}\right)_{d}=0$, then $\operatorname{dim}\left(I_{X^{\prime \prime}}\right)_{d}=0$, for any subscheme $X^{\prime \prime} \supset X$.

Proof. (i)

$$
\begin{aligned}
\mathrm{HF}(X, d) & =\sum_{i=1}^{s} \operatorname{HF}\left(X_{i}, d\right)=\sum_{i=1}^{s^{\prime}} \operatorname{HF}\left(X_{i}, d\right)+\sum_{i=s^{\prime}+1}^{s} \operatorname{HF}\left(X_{i}, d\right) \\
& \geq \operatorname{HF}\left(X^{\prime}, d\right)+\sum_{i=s^{\prime}+1}^{s} \operatorname{HF}\left(X_{i}, d\right) \geq \operatorname{HF}(X, d) .
\end{aligned}
$$

Hence the inequalites are equalities, and we get the conclusion.

(ii) Since $\operatorname{HF}(X, d)=\operatorname{HF}(Y, d)+\left(\begin{array}{c}m+n-1 \\ n\end{array}\right)$, and $I_{X}=I_{Y} \cap I_{m P}$, from the exact sequence

$$
0 \longrightarrow R / I_{Y} \cap I_{m P} \longrightarrow R / I_{Y} \oplus R / I_{m P} \longrightarrow R /\left(I_{Y}+I_{m P}\right) \longrightarrow 0
$$

we get that $\operatorname{dim}\left(R /\left(I_{Y}+I_{m P}\right)\right)_{d}=0$ and $\operatorname{HF}(m P, d)=\left(\begin{array}{c}m+n-1 \\ n\end{array}\right)$. It follows that $\operatorname{dim}\left(R /\left(I_{Y}+I_{m^{\prime} P}\right)\right)_{d}=0$ and $\operatorname{HF}\left(m^{\prime} P, d\right)=\left(\begin{array}{c}m^{\prime}+n-1 \\ n\end{array}\right)$. Thus from the analogous sequence for $m^{\prime} P$ we get

$$
\operatorname{HF}\left(X^{\prime}, d\right)=\operatorname{HF}(Y, d)+\operatorname{HF}\left(m^{\prime} P, d\right)=\operatorname{HF}(Y, d)+\left(\begin{array}{c}
m^{\prime}+n-1 \\
n
\end{array}\right) .
$$

(iii) Obvious. 
We now recall some basic notions contained in [4].

Definition 2.4. We say that $C$ is a degenerate conic if $C$ is the union of two intersecting lines $L, M$. In this case we write $C=L+M$.

Definition 2.5. Let $L$ and $M$ be two intersecting lines in $\mathbb{P}^{n}(n \geq 3)$, let $P=L \cap M$, and let $T \simeq \mathbb{P}^{3}$ be a generic linear space containing the scheme $L+M$. We call the scheme $L+M+\left.2 P\right|_{T}$ a degenerate conic with an embedded point or a 3-dimensional sundial (see [13], or [4, Definition 3.7]).

Remark 2.6. A degenerate conic with an embedded point can be viewed either as a degeneration of two generic lines, or as a degeneration of a scheme which is the union of a degenerate conic and a simple generic point (see [4, Lemma 3.8 and Remark 3.9]).

We refer the reader to [4, Theorem 4.4] for a proof of the following technical result.

Theorem 2.7. Let $n \geq 3$ and let $X \subset \mathbb{P}^{n}$ be the union of $s$ generic 3-dimensional sundials and $l$ generic lines. Then $X$ has bipolynomial Hilbert function, that is,

$$
\operatorname{HF}(X, d)=\min \left\{\left(\begin{array}{c}
d+n \\
n
\end{array}\right) ;(d+1)(2 s+l)\right\} .
$$

Equivalently, the following schemes have the expected Hilbert Function in degree $d$ :

$$
\begin{aligned}
W & = \begin{cases}\widehat{C}_{1}+\cdots+\widehat{C}_{s}+P_{1}+\cdots+P_{r} & \text { for } t \text { even } \\
\widehat{C}_{1}+\cdots+\widehat{C}_{s}+M+P_{1}+\ldots P_{r} & \text { for } t \text { odd },\end{cases} \\
T & = \begin{cases}\widehat{C}_{1}+\cdots+\widehat{C}_{s}+M & \text { for } t \text { even and } r>0 \\
\widehat{C}_{1}+\cdots+\widehat{C}_{s+1} & \text { for } t \text { odd } \text { and } r>0,\end{cases}
\end{aligned}
$$

where

$$
t=\left\lfloor\frac{\left(\begin{array}{c}
d+n \\
n
\end{array}\right)}{d+1}\right\rfloor, \quad r=\left(\begin{array}{c}
d+n \\
n
\end{array}\right)-t(d+1), \quad s=\left\lfloor\frac{t}{2}\right\rfloor,
$$

and the $\widehat{C}_{i}$ 's are degenerate conics with an embedded point, that is 3-dimensional sundials, the $P_{i}$ 's are generic points, and $M$ is a generic line, that is,

$$
\begin{aligned}
\operatorname{dim}\left(I_{W}\right)_{d} & =\exp \operatorname{dim}\left(I_{W}\right)_{d}=\left(\begin{array}{c}
d+n \\
n
\end{array}\right)-t(d+1)-r=0, \\
\operatorname{dim}\left(I_{T}\right)_{d} & =\exp \operatorname{dim}\left(I_{W}\right)_{d}=\max \left\{\left(\begin{array}{c}
d+n \\
n
\end{array}\right)-(t+1)(d+1) ; 0\right\}=0 .
\end{aligned}
$$




\section{The main theorem in $\mathbb{P}^{n}$, for $n \geq 4$}

In this section we will prove (see Theorem 3.2) that for $n \geq 4$, the ideal of the scheme $X \subset \mathbb{P}^{n}$ consisting of $s$ generic lines and a generic point of multiplicity $m$ has the expected dimension. We start with the following proposition, which, for $m \leq d$, is equivalent to Theorem 3.2.

Proposition 3.1. Let $n, d, m \in \mathbb{N}$, with $n \geq 4$ and $m \leq d$. Let

$$
e=\left\lfloor\frac{\left(\begin{array}{c}
d+n \\
n
\end{array}\right)-\left(\begin{array}{c}
m+n-1 \\
n
\end{array}\right)}{d+1}\right\rfloor, \quad r=\left(\begin{array}{c}
d+n \\
n
\end{array}\right)-\left(\begin{array}{c}
m+n-1 \\
n
\end{array}\right)-e(d+1)
$$

The ideal of the scheme $X \subset \mathbb{P}^{n}$ consisting of e generic lines $L_{1}, \ldots, L_{e}$ and together with $r$ generic points $P_{1}, \ldots, P_{r}$ lying on a generic line L, together with a generic point $P$ of multiplicity $m$, has the expected dimension, that is,

$$
\operatorname{dim}\left(I_{X}\right)_{d}=\left(\begin{array}{c}
d+n \\
n
\end{array}\right)-\left(\begin{array}{c}
m+n-1 \\
n
\end{array}\right)-e(d+1)-r=0 .
$$

Proof. We will prove the proposition by induction on $d-m$.

Let $d=m$. Since for $d=m$ any form of degree $d$ in $I_{X}$ represents a cone with $P$ as its vertex, it follows that

$$
\operatorname{dim}\left(I_{X}\right)_{d}=\operatorname{dim}\left(I_{W}\right)_{d}
$$

where $W \subset \mathbb{P}^{n-1}$ consists of $e$ generic lines and $r$ generic points lying on a line. Since for $d=m$ we have $\left(\begin{array}{c}d+n \\ n\end{array}\right)-\left(\begin{array}{c}m+n-1 \\ n\end{array}\right)=\left(\begin{array}{c}d+n-1 \\ n-1\end{array}\right)$, we get

$$
e=\left\lfloor\frac{\left(\begin{array}{c}
d+n-1 \\
n-1
\end{array}\right)}{d+1}\right\rfloor, \quad r=\left(\begin{array}{c}
d+n-1 \\
n-1
\end{array}\right)-e(d+1) .
$$

So by [13, Theorem 0.2] we get

$$
\operatorname{dim}\left(I_{W}\right)_{d}=\left(\begin{array}{c}
d+n-1 \\
n-1
\end{array}\right)-e(d+1)-r=0
$$

and we are done for $m=d$.

Assume $m<d$. Let

$$
\begin{aligned}
e^{\prime} & =\left\lfloor\frac{\left(\begin{array}{c}
d-1+n \\
n
\end{array}\right)-\left(\begin{array}{c}
m+n-1 \\
n
\end{array}\right)-r}{d}\right\rfloor, \\
r^{\prime} & =\left(\begin{array}{c}
d-1+n \\
n
\end{array}\right)-\left(\begin{array}{c}
m+n-1 \\
n
\end{array}\right)-r-e^{\prime} d .
\end{aligned}
$$


Since $\left(\begin{array}{c}d-1+n \\ n\end{array}\right)-\left(\begin{array}{c}m+n-1 \\ n\end{array}\right)-r \geq 0$, we have $e^{\prime} \geq 0$ (for a proof of this inequality see [5, Lemma 5.1 (i)], a preliminary version on-line of the present paper).

Notice that $e-e^{\prime}-2 r^{\prime} \geq 0$ (this inequality is treated in [5, Lemma 5.1 (ii)]). Using this inequality we construct a scheme $Y$ obtained from $X$ by specializing some lines and by degenerating other pairs of lines into a hyperplane $H \simeq \mathbb{P}^{n-1}$.

More precisely, we specialize $e-e^{\prime}-2 r^{\prime}$ lines into $H$ and we degenerate $r^{\prime}$ pairs of lines in order to obtain the following specialization of $X$ :

$Y=\widehat{C}_{1}+\cdots+\widehat{C}_{r^{\prime}}+M_{1}+\cdots+M_{e-e^{\prime}-2 r^{\prime}}+L_{1}+\cdots+L_{e^{\prime}}+m P+P_{1}+\cdots+P_{r} ;$

here the $M_{i} \subset H$ are generic lines and the $\widehat{C}_{i} \subset H_{i} \simeq \mathbb{P}^{3}$ are 3-dimensional sundials such that $\widehat{C}_{i}$ is the union of a degenerate conic $C_{i}$ lying on $H$ and a double point $\left.2 Q_{i}\right|_{H_{i}} \not \subset H$.

So we have

$\operatorname{Res}_{H} Y=Q_{1}+\cdots+Q_{r^{\prime}}+L_{1}+\cdots+L_{e^{\prime}}+m P+P_{1}+\cdots+P_{r} \subset \mathbb{P}^{n}$, $\operatorname{Tr}_{H} Y=C_{1}+\cdots+C_{r^{\prime}}+M_{1}+\cdots+M_{e-e^{\prime}-2 r^{\prime}}+T_{1}+\cdots+T_{e^{\prime}} \subset H \simeq \mathbb{P}^{n-1}$, where $T_{i}=L_{i} \cap H$ and the $T_{i}$ are generic points.

Since $e^{\prime} \geq r^{\prime}$ (this inequality is proved in [5, Lemma 5.1 (iii)]), and $r^{\prime} \leq$ $d-1$, by Remark 2.6, Lemma 2.3 and Theorem 2.7, we get that the dimension of $\operatorname{dim}\left(I_{\operatorname{Tr}_{H} Y}\right)_{d}$ is as expected, that is,

$$
\begin{aligned}
\operatorname{dim}\left(I_{\operatorname{Tr}_{H} Y}\right)_{d} & =\left(\begin{array}{c}
d+n-1 \\
n-1
\end{array}\right)-r^{\prime}(2 d+1)-\left(e-e^{\prime}-2 r^{\prime}\right)(d+1)-e^{\prime} \\
& =\left(\begin{array}{c}
d+n-1 \\
n-1
\end{array}\right)-\left(e-e^{\prime}\right)(d+1)+r^{\prime}-e^{\prime} \\
& =\left(\begin{array}{c}
d+n-1 \\
n-1
\end{array}\right)-e(d+1)+\left(\begin{array}{c}
d-1+n \\
n
\end{array}\right)-\left(\begin{array}{c}
m+n-1 \\
n
\end{array}\right)-r \\
& =\left(\begin{array}{c}
d+n-1 \\
n-1
\end{array}\right)-e(d+1)+\left(\begin{array}{c}
d-1+n \\
n
\end{array}\right)-\left(\begin{array}{c}
d+n \\
n
\end{array}\right)+e(d+1)=0 .
\end{aligned}
$$

Now we compute the dimension of the residue. Let

$$
\operatorname{Res}_{H} Y=Y_{1}+Y_{2} \text {, }
$$

where

$$
\begin{aligned}
& Y_{1}=Q_{1}+\cdots+Q_{r^{\prime}}+L_{1}+\cdots+L_{e^{\prime}}+m P, \\
& Y_{2}=P_{1}+\cdots+P_{r} \subset L .
\end{aligned}
$$

By the inductive hypothesis and since $r \leq d$ we have

$$
\begin{aligned}
\operatorname{dim}\left(I_{Y_{1}}\right)_{d-1}= & \left(\begin{array}{c}
d-1+n \\
n
\end{array}\right)-r^{\prime}-e^{\prime} d-\left(\begin{array}{c}
m+n-1 \\
n
\end{array}\right) \\
= & \left(\begin{array}{c}
d-1+n \\
n
\end{array}\right)-\left(\begin{array}{c}
d-1+n \\
n
\end{array}\right)+\left(\begin{array}{c}
m+n-1 \\
n
\end{array}\right)+r \\
& +e^{\prime} d-e^{\prime} d-\left(\begin{array}{c}
m+n-1 \\
n
\end{array}\right)=r
\end{aligned}
$$


and

$$
\operatorname{dim}\left(I_{Y_{1}+L}\right)_{d-1}=\max \{r-d ; 0\}=0 .
$$

Hence, by [4, Lemma 3.5] we get

$$
\operatorname{dim}\left(I_{\operatorname{Res}_{H}} Y\right)_{d-1}=0 .
$$

Now, since $\operatorname{dim}\left(I_{\operatorname{Tr}_{H} Y}\right)_{d}=\operatorname{dim}\left(I_{\operatorname{Res}_{H} Y}\right)_{d-1}=0$, by Castelnuovo's Inequality (see Lemma 2.2) the conclusion follows.

Theorem 3.2. Let $n, d, s, m \in \mathbb{N}$. For $n \geq 4$, the ideal of the scheme $X \subset \mathbb{P}^{n}$ consisting of $s$ generic lines $L_{1}, \ldots, L_{s}$ and a generic point $P$ of multiplicity $m$ has the expected dimension, that is,

$$
\operatorname{dim}\left(I_{X}\right)_{d}=\max \left\{\left(\begin{array}{c}
d+n \\
n
\end{array}\right)-\left(\begin{array}{c}
m+n-1 \\
n
\end{array}\right)-s(d+1), 0\right\} .
$$

Proof. Obvious for $m>d$, so assume that $m \leq d$.

For $\left(\begin{array}{c}d+n \\ n\end{array}\right)-\left(\begin{array}{c}m+n-1 \\ n\end{array}\right)-s(d+1) \geq 0$ the conclusion follows from Proposition 3.1 and Lemma 2.3 (i)

$$
\begin{aligned}
& \text { If }\left(\begin{array}{c}
d+n \\
n
\end{array}\right)-\left(\begin{array}{c}
m+n-1 \\
n
\end{array}\right)-s(d+1)<0 \text {, let } e, r \text { be as in Proposition 3.1, hence } \\
& s(d+1)>\left(\begin{array}{c}
d+n \\
n
\end{array}\right)-\left(\begin{array}{c}
m+n-1 \\
n
\end{array}\right)=e(d+1)+r, \quad 0 \leq r<d+1 .
\end{aligned}
$$

It follows that $s>e$. Now consider a subscheme $Y \subset X$ consisting of the lines $L_{1}, \ldots, L_{e}$ and $r$ generic points lying on the line $L_{e+1}$. Since, by Proposition 3.1, $\operatorname{dim}\left(I_{Y}\right)_{d}=0$, then $\operatorname{dim}\left(I_{X}\right)_{d}=0$ follows.

\section{The main theorem in $\mathbb{P}^{3}$}

Proposition 4.1. Let $d \in \mathbb{N}$, with $d \geq 3$. Let

$$
e=\left\lfloor\frac{\left(\begin{array}{c}
d+3 \\
3
\end{array}\right)-4}{d+1}\right\rfloor, \quad r=\left(\begin{array}{c}
d+3 \\
3
\end{array}\right)-4-e(d+1) .
$$

The ideal of the scheme $X \subset \mathbb{P}^{3}$ consisting of e generic lines $L_{1}, \ldots, L_{e}$ and together with $r$ generic points $P_{1}, \ldots, P_{r}$ together with a generic double point supported on $P$, has the expected dimension, that is,

$$
\operatorname{dim}\left(I_{X}\right)_{d}=\left(\begin{array}{c}
d+3 \\
3
\end{array}\right)-4-e(d+1)-r=0
$$


Proof. We will prove the theorem by induction on $d$.

For $d=3$ we have $e=4$ and $r=0$ so

$$
X=2 P+L_{1}+\cdots+L_{4} .
$$

Since the trace of $X$ on the plane $\left\langle P, L_{i}\right\rangle$ is formed by the line $L_{i}$, one double point and three simple points, then the surfaces defined by the forms of degree 3 in $I_{X}$ have the plane $\left\langle P, L_{i}\right\rangle$ as a fixed component. But the four planes $\left\langle P, L_{i}\right\rangle$ cannot be fixed components for a surface of degree 3. It follows that $\operatorname{dim}\left(I_{X}\right)_{3}=0$.

For $d=4$ we have $e=6$ and $r=1$ so

$$
X=2 P+L_{1}+\cdots+L_{6}+P_{1} .
$$

Now we degenerate the scheme $X$ : first we degenerate the lines $L_{1}$ and $L_{2}$, so that they become a 3 -dimensional sundial $\widehat{C}$, then we specialize the line $L_{3}$ on the plane $H=\left\langle P, R, P_{1}\right\rangle$, where $R$ is the double point of $\widehat{C}$. Let

$$
\widetilde{X}=2 P+\widehat{C}+L_{3}+\cdots+L_{6}+P_{1}
$$

be the degenerate scheme.

The trace of $\widetilde{X}$ on the plane $H$ is

$$
\operatorname{Tr}_{H} \tilde{X}=2 P|H+2 R|_{H}+L_{3}+P_{1}+\left(L_{4}+L_{5}+L_{6}\right) \cap H \subset H \simeq \mathbb{P}^{2},
$$

hence

$$
\operatorname{dim}\left(I_{\operatorname{Tr}_{H}} \widetilde{X}\right)_{4}=\operatorname{dim}\left(I_{\operatorname{Tr}_{H}} \widetilde{X}-L_{3}\right)_{3} .
$$

Since $\left(\operatorname{Tr}_{H} \tilde{X}-L_{3}\right)$ is the union of two double points and four simple points, it follows that $\operatorname{dim}\left(I_{\operatorname{Tr}_{H}} \tilde{X}\right)_{4}=0$. So $H$ is a fixed component for the forms of $\left(I_{\tilde{X}}\right)_{4}$, and we have

$$
\operatorname{dim}\left(I_{\widetilde{X}}\right)_{4}=\operatorname{dim}\left(I_{\operatorname{Res}_{H}} \tilde{X}\right)_{3},
$$

where $\operatorname{Res}_{H} \tilde{X}$ is the union of three lines, a point and a degenerate conic $C$, say

$$
\operatorname{Res}_{H} \tilde{X}=P+C+L_{4}+L_{5}+L_{6} .
$$

Now, if we degenerate $P$ and $C$, we obtain again the sundial $\widehat{C}$, so, by Theorem 2.7 we have

$$
\operatorname{dim}\left(I_{\operatorname{Res}_{H}} \tilde{X}\right)_{3}=0,
$$

and from here we get $\operatorname{dim}\left(I_{X}\right)_{4}=0$.

Now let $d \geq 5$. Let $Q$ be a smooth quadric: we will specialize some of the lines of the scheme $X$ on $Q$. We consider three cases.

Case 1: $d \equiv 0 \bmod 3$. Let $d=3 h$. Note that, since $d \geq 5$, then $h \geq 2$. We have:

$$
\begin{aligned}
e & =\frac{(h+1)(3 h+2)}{2}-1, \quad r=3(h-1) \geq 3, \\
X & =2 P+L_{1}+\ldots+L_{e}+P_{1}+\ldots .+P_{r} .
\end{aligned}
$$


Let $\tilde{X}$ be the scheme obtained from $X$ by specializing $2 h+1$ lines in such a way that the lines $L_{1}, \ldots, L_{2 h+1}$ become lines of the same ruling on $Q$, (the lines $L_{2 h+2}, \ldots, L_{e}$ remain generic lines, not lying on $Q$ ), and by specializing on $Q$ the points $P_{1}$ and $P_{2}$. We have

$$
\operatorname{Res}_{Q} \tilde{X}=2 P+L_{2 h+2}+\ldots+L_{e}+P_{3}+\ldots .+P_{r} .
$$

By the inductive hypothesis we have:

$$
\begin{aligned}
\operatorname{dim}\left(I_{\operatorname{Res}_{Q} \tilde{X}}\right)_{d-2}= & \left(\begin{array}{c}
3 h+1 \\
3
\end{array}\right)-4-(e-2 h-1)(3 h-1)-(r-2) \\
= & \frac{h(3 h+1)(3 h-1)}{2}-4-\frac{(h+1)(3 h-2)}{2}(3 h-1) \\
& -(3 h-5)=0 .
\end{aligned}
$$

Now

$$
\operatorname{Tr}_{Q} \tilde{X}=L_{1}+\cdots+L_{2 h+1}+\operatorname{Tr}_{Q}\left(L_{2 h+2}+\ldots+L_{e}\right)+P_{1}+P_{2} .
$$

Since the trace on $Q$ of the $(e-2 h-1)$ lines $L_{2 h+2}, \ldots, L_{e}$ consists of $2(e-2 h-1)$ generic points, we have that $\operatorname{Tr}_{Q} \widetilde{X}$ consists of $(2 h+1)$ lines of the same ruling, and $(2 e-4 h)$ generic points. Thinking of $Q$ as $\mathbb{P}^{1} \times \mathbb{P}^{1}$, we see that the forms of degree $3 h$ in the ideal of $\operatorname{Tr}_{Q} \widetilde{X}$ are curves of type $(3 h-(2 h+1), 3 h)=(h-1,3 h)$ in $\mathbb{P}^{1} \times \mathbb{P}^{1}$ passing through $(2 e-4 h)$ generic points. Hence

$$
\operatorname{dim}\left(I_{\operatorname{Tr}_{Q}} \tilde{X}\right)_{3 h}=h(3 h+1)-2 e+4 h=0 .
$$

So by Lemma 2.2 and by the semicontinuity of the Hilbert function we get $\operatorname{dim}\left(I_{X}\right)_{3 h}=0$.

Case 2: $d \equiv 2 \bmod 3$. For the computation in this case, recall that we will think of $Q$ as $\mathbb{P}^{1} \times \mathbb{P}^{1}$ and that (see, for instance, [7, Section 2]) in the case we are treating each of the double points on $Q$ will give three independent condition to our forms.

Let $d=3 h+2$. We have:

$$
\begin{aligned}
& \text { for } h=1: \quad d=5 \quad ; \quad e=8 \quad ; \quad r=4 \quad \text {; } \\
& \text { for } h=2: \quad d=8 \quad ; \quad e=17 \quad ; \quad r=8 \quad \text {; } \\
& \text { for } h \geq 3: \quad d=3 h+2 ; \quad e=\frac{3(h+1)(h+2)}{2} ; \quad r=h-3 \text {. }
\end{aligned}
$$

For $h=1$, we have

$$
X=2 P+L_{1}+\cdots+L_{8}+P_{1}+\cdots+P_{4} .
$$

Specialize the scheme $X$ in such a way that the lines $L_{1}, \ldots, L_{4}$ become lines of the same ruling on $Q$, and the points $P$ and $P_{1}$ become points on $Q$. We get

$$
\begin{aligned}
\operatorname{Res}_{Q} \tilde{X} & =P+L_{5}+\cdots+L_{8}+P_{2}+\cdots+P_{4}, \\
\operatorname{Tr}_{Q} \tilde{X} & =\left.2 P\right|_{Q}+L_{1}+\cdots+L_{4}+\operatorname{Tr}_{Q}\left(L_{5}+\ldots+L_{8}\right)+P_{1},
\end{aligned}
$$


and we easily get

$$
\begin{gathered}
\operatorname{dim}\left(I_{\operatorname{Res}_{Q} \tilde{X}}\right)_{3}=20-1-16-3=0, \\
\operatorname{dim}\left(I_{\operatorname{Tr}_{Q}} \tilde{X}\right)_{5}=12-3-8-1=0 .
\end{gathered}
$$

For $h=2$, we have

$$
X=2 P+L_{1}+\cdots+L_{17}+P_{1}+\cdots+P_{8} .
$$

We specialize the scheme $X$ so that the lines $L_{1}, \ldots, L_{6}$ become lines of the same ruling on $Q$, and the points $P, P_{1}$ and $P_{2}$ become points on $Q$. We get

$$
\begin{aligned}
\operatorname{Res}_{Q} \tilde{X} & =P+L_{7}+\cdots+L_{17}+P_{3}+\cdots+P_{8}, \\
\operatorname{Tr}_{Q} \tilde{X} & =\left.2 P\right|_{Q}+L_{1}+\cdots+L_{6}+\operatorname{Tr}_{Q}\left(L_{7}+\ldots+L_{17}\right)+P_{1}+P_{2},
\end{aligned}
$$

and we have

$$
\begin{gathered}
\operatorname{dim}\left(I_{\operatorname{Res}_{Q} \tilde{X}}\right)_{6}=84-1-77-6=0, \\
\operatorname{dim}\left(I_{\operatorname{Tr}_{Q} \tilde{X}}\right)_{8}=27-3-22-2=0 .
\end{gathered}
$$

For $h \geq 3$, we have

$$
X=2 P+L_{1}+\cdots+L_{e}+P_{1}+\cdots+P_{h-3} .
$$

Now we degenerate the lines $L_{1}$ and $L_{2}$, so that they become a 3-dimensional sundial $\widehat{C}=C+2 R$, where $\mathrm{C}$ is a degenerate conic and $2 R$ is a double point. Then we specialize the points $R, P, P_{1} \ldots P_{h-3}$ so that they become points on $Q$, and the lines $L_{3}, \ldots, L_{2 h+4}$ so that they become lines of the same ruling on $Q$. Let $\widetilde{X}$ be the specialized scheme. We have

$$
\operatorname{Res}_{Q} \tilde{X}=P+C+L_{2 h+5}+\cdots+L_{e}
$$

and, by Remark 2.6, we get

$$
\operatorname{dim}\left(I_{\operatorname{Res}_{Q} \tilde{X}}\right)_{3 h}=\left(\begin{array}{c}
3 h+3 \\
3
\end{array}\right)-2(3 h+1)-(e-2 h-4)(3 h+1)=0 .
$$

Moreover

$$
\begin{aligned}
\operatorname{Tr}_{Q} \tilde{X}= & \left.2 P\right|_{Q}+\left.2 R\right|_{Q}+L_{3}+\cdots+L_{2 h+4}+\operatorname{Tr}_{Q}\left(L_{2 h+5}+\ldots+L_{e}\right) \\
& +P_{1}+\cdots+P_{h-3},
\end{aligned}
$$

and we get

$\operatorname{dim}\left(I_{\operatorname{Tr}_{Q}} \tilde{X}\right)_{3 h+2}=(h+1)(3 h+3)-3-3-2-2(e-2 h-5+1)-(h-3)=0$.

So by Lemma 2.2 and by the semicontinuity of the Hilbert function we get $\operatorname{dim}\left(I_{X}\right)_{3 h+2}=0$. 
Case 3: $d \equiv 1 \bmod 3$. Let $d=3 h+1$. Note that $h \geq 2$. We have

$$
e=\frac{(h+1)(3 h+4)}{2}-1, \quad r=3 h-2 .
$$

We specialize the scheme $X$ in such a way that the lines $L_{1}, \ldots, L_{2 h+1}$ become lines of the same ruling on $Q$, and the points $P$ and $P_{1}, \ldots, P_{2 h-1}$ become points on $Q$. Let $\widetilde{X}$ be the specialized scheme. So

$$
\operatorname{Res}_{Q} \tilde{X}=P+L_{2 h+2}+\ldots+L_{e}+P_{2 h}+\ldots .+P_{3 h-2},
$$

and by [13, Theorem 0.1$]$ we have

$$
\begin{aligned}
\operatorname{dim}\left(I_{\operatorname{Res}_{Q} \widetilde{X}}\right)_{3 h-1} & =\left(\begin{array}{c}
3 h+2 \\
3
\end{array}\right)-1-3 h(e-2 h-1)-(h-1) \\
& =\frac{h(3 h+2)(3 h+1)}{2}-1-\frac{9 h^{2}(h+1)}{2}-h+1=0 .
\end{aligned}
$$

The trace of $\tilde{X}$ on $Q$ consists of the $(2 h+1)$ lines of the same ruling $L_{1}, \ldots, L_{2 h+1}$, the double point $P$, the simple points $P_{1}, \ldots, P_{2 h-1}$, and the trace of the lines $L_{2 h+2}, \ldots, L_{e}$. As usual, thinking of $Q$ as $\mathbb{P}^{1} \times \mathbb{P}^{1}$, we see that the forms of degree $3 h+1$ in the ideal of $\operatorname{Tr}_{Q} \widetilde{X}$ are curves of type $((3 h+1)-(2 h+1), 3 h+1)=$ $(h, 3 h+1)$ in $\mathbb{P}^{1} \times \mathbb{P}^{1}$. Hence, since it is easy to prove that the double point $P$ gives 3 independent conditions to our forms (see, for instance, [7, Section 2]), we have

$$
\operatorname{dim}\left(I_{\operatorname{Tr}_{Q}} \tilde{X}\right)_{3 h+1}=(h+1)(3 h+2)-3-(2 h-1)-2(e-2 h-1)=0 .
$$

So also in this case, by Lemma 2.2 and by the semicontinuity of the Hilbert function, we get $\operatorname{dim}\left(I_{X}\right)_{3 h+1}=0$.

Theorem 4.2. Let $d, s, m \in \mathbb{N}$, with $d \geq 1$. Let $X \subset \mathbb{P}^{3}$ be the scheme consisting of $s \geq 1$ generic lines and a generic point $P$ of multiplicity $m \geq 1$.

(i) The ideal of $X \subset \mathbb{P}^{3}$ has the expected dimension, that is,

$$
\operatorname{dim}\left(I_{X}\right)_{d}=\exp \operatorname{dim}\left(I_{X}\right)_{d}=\max \left\{\left(\begin{array}{c}
d+3 \\
3
\end{array}\right)-\left(\begin{array}{c}
m+2 \\
3
\end{array}\right)-s(d+1), 0\right\},
$$

(a) for $m>d$, any $s$;

(b) for $m=d$ and $s>d$, or for $m=d$ and $s=1$;

(c) for $m=d-1$, any $s$;

(d) for $m<d-1$ and $1 \leq s \leq m+2$;

(e) for $m=2, d \geq 3$, any $s$;

(f) for $m=1$, any $d$, and any $s$. 
(ii) For $m=d \geq 2$ and $2 \leq s \leq d$, the dimension of $\left(I_{X}\right)_{d}$ is

$$
\operatorname{dim}\left(I_{X}\right)_{d}=\left(\begin{array}{c}
d-s+2 \\
2
\end{array}\right) \neq \exp \operatorname{dim}\left(I_{X}\right)_{d}
$$

and the defect is

$$
\delta=\left\{\begin{array}{lll}
\left(\begin{array}{l}
s \\
2
\end{array}\right) & \text { for } & s \leq \frac{d+2}{2} \\
\left(\begin{array}{c}
d-s+2 \\
2
\end{array}\right) & \text { for } & \frac{d+2}{2} \leq s \leq d .
\end{array}\right.
$$

Proof. (i) (a) Obvious. We have $\operatorname{dim}\left(I_{X}\right)_{d}=\exp \operatorname{dim}\left(I_{X}\right)_{d}=0$.

(i) (b) and (ii). If $m=d$ any form of degree $d$ in $I_{X}$ represents a cone whose vertex contains $P$. Hence

$$
\operatorname{dim}\left(I_{X}\right)_{d}=\operatorname{dim}\left(I_{X^{\prime}}\right)_{d},
$$

where $X^{\prime} \subset \mathbb{P}^{2}$ is the projection of $X$ from $P$ in a $\mathbb{P}^{2}$ and it is a scheme consisting of $s$ generic lines. Hence, for $s>d$, we immediately get $\operatorname{dim}\left(I_{X}\right)_{d}=0$.

For $s \leq d$ we have

$$
\operatorname{dim}\left(I_{X}\right)_{d}=\left(\begin{array}{c}
d-s+2 \\
2
\end{array}\right)
$$

Since in this case the expected dimension of $\left(I_{X}\right)_{d}$ is

$$
\begin{aligned}
& \exp \operatorname{dim}\left(I_{X}\right)_{d}=\max \left\{\left(\begin{array}{c}
d+3 \\
3
\end{array}\right)-\left(\begin{array}{c}
d+2 \\
3
\end{array}\right)-s(d+1), 0\right\} \\
& = \begin{cases}\left(\begin{array}{c}
d+2 \\
2
\end{array}\right)-s(d+1) & \text { for } s \leq \frac{d+2}{2} \\
0 & \text { for } s \geq \frac{d+2}{2}\end{cases}
\end{aligned}
$$

then for $s=1$ we have $\operatorname{dim}\left(I_{X}\right)_{d}=\exp \operatorname{dim}\left(I_{X}\right)_{d}$, and so we are done with (i)(b).

For $2 \leq s \leq d$ the defect is

$$
\operatorname{dim}\left(I_{X}\right)_{d}-\exp \operatorname{dim}\left(I_{X}\right)_{d}= \begin{cases}\left(\begin{array}{l}
s \\
2
\end{array}\right) & \text { for } s \leq \frac{d+2}{2} \\
\left(\begin{array}{c}
d-s+2 \\
2
\end{array}\right) & \text { for } s \geq \frac{d+2}{2}\end{cases}
$$

so we have proved (ii). 
(i) (c). By induction on $d$. Obvious for $d=1$, let $d>1$. Let

$$
X=L_{1}+\cdots+L_{s}+m P
$$

be our scheme, where the $L_{i}$ are generic lines. Since $d=m+1$, we have that

$$
\begin{aligned}
\exp \operatorname{dim}\left(I_{X}\right)_{d} & =\max \left\{\left(\begin{array}{c}
d+3 \\
3
\end{array}\right)-\left(\begin{array}{c}
d+1 \\
3
\end{array}\right)-s(d+1) ; 0\right\} \\
& =\max \left\{(d+1)^{2}-s(d+1) ; 0\right\},
\end{aligned}
$$

hence it is enough to prove that $\left(I_{X}\right)_{d}$ has the expected dimension for $s=d+1$, and the conclusion will follows from Lemma 2.3.

Let $H \simeq \mathbb{P}^{2}$ be the plane though $P$ and $L_{1}$. The trace of $X$ on $H$ is

$$
\operatorname{Tr}_{H} X=\left.m P\right|_{H}+L_{1}+R_{2}+\cdots+R_{d+1},
$$

where $R_{i}=L_{i} \cap H$, and the $R_{i}$ are $d$ generic points on $H$.

Since $L_{1}$ is a fixed component for the curves defined by the forms of $I_{\operatorname{Tr}_{H} X}$, we have

$$
\begin{aligned}
\operatorname{dim}\left(I_{\operatorname{Tr}_{H} X}\right)_{d} & =\operatorname{dim}\left(I_{\operatorname{Tr}_{H} X-L_{1}}\right)_{d-1}=\left(\begin{array}{c}
d+1 \\
2
\end{array}\right)-\left(\begin{array}{c}
m+1 \\
2
\end{array}\right)-d \\
& =\left(\begin{array}{c}
d+1 \\
2
\end{array}\right)-\left(\begin{array}{l}
d \\
2
\end{array}\right)-d=0 .
\end{aligned}
$$

It follows that $H$ is a fixed component for the forms of $\left(I_{X}\right)_{d}$, so

$$
\operatorname{dim}\left(I_{X}\right)_{d}=\operatorname{dim}\left(I_{\operatorname{Res}_{H} X}\right)_{d-1}
$$

where

$$
\operatorname{Res}_{H} X=(m-1) P+L_{2}+\cdots+L_{s}=(d-2) P+L_{2}+\cdots+L_{d+1} .
$$

By the inductive hypothesis we get

$$
\operatorname{dim}\left(I_{\operatorname{Res}_{H} X}\right)_{d-1}=\left(\begin{array}{c}
d+2 \\
3
\end{array}\right)-\left(\begin{array}{l}
d \\
3
\end{array}\right)-d^{2}=0,
$$

and we are done with (i) (c).

(i) (d). Since for $m=d-1$, and $s=m+2$ by (i) (c) we have

$$
\operatorname{dim}\left(I_{X}\right)_{d}=\left(\begin{array}{c}
d+3 \\
3
\end{array}\right)-\left(\begin{array}{c}
m+2 \\
3
\end{array}\right)-s(d+1),
$$

by Lemma 2.3 (i) and (ii) we get the conclusion. 
(i)(e). Let $m=2$ and $d \geq 3$. We have to prove that

$$
\operatorname{dim}\left(I_{X}\right)_{d}=\exp \operatorname{dim}\left(I_{X}\right)_{d}=\max \left\{\left(\begin{array}{c}
d+3 \\
3
\end{array}\right)-4-s(d+1), 0\right\} .
$$

If $\left(\begin{array}{c}d+3 \\ 3\end{array}\right)-4-s(d+1) \geq 0$, let

$$
e=\left\lfloor\frac{\left(\begin{array}{c}
d+3 \\
3
\end{array}\right)-4}{d+1}\right\rfloor, \quad r=\left(\begin{array}{c}
d+3 \\
3
\end{array}\right)-4-e(d+1),
$$

and let $P_{1}, \ldots, P_{r}$ be generic points.

By Proposition 4.1 we know that for $s=e$

$$
\operatorname{dim}\left(I_{X+P_{1}+\cdots+P_{r}}\right)_{d}=0
$$

hence for $s=e$ we have

$$
\operatorname{dim}\left(I_{X}\right)_{d}=r=\exp \operatorname{dim}\left(I_{X}\right)_{d}
$$

and now the conclusion follows from Lemma 2.3 (i). Now let

$$
\left(\begin{array}{c}
d+3 \\
3
\end{array}\right)-4-s(d+1)<0
$$

In this case we have

$$
s>\frac{\left(\begin{array}{c}
d+3 \\
3
\end{array}\right)-4}{(d+1)}= \begin{cases}\frac{(h+1)(3 h+2)}{2}-\frac{4}{3 h+1} & \text { for } \quad d=3 h \\
\frac{(h+1)(3 h+4)}{2}-\frac{4}{3 h+2} & \text { for } \quad d=3 h+1 \\
\frac{3(h+1)(h+2)}{2}+\frac{h-3}{3 h+3} & \text { for } \quad d=3 h+2,\end{cases}
$$

that is,

$$
s \geq \begin{cases}\frac{(h+1)(3 h+2)}{2} & \text { for } \quad d=3 h ; \\ \frac{(h+1)(3 h+4)}{2} & \text { for } \quad d=3 h+1 \\ 9 & \text { for } \quad d=5 \\ 18 & \text { for } \quad d=8 \\ \frac{3(h+1)(h+2)}{2}+1 & \text { for } \quad d=3 h+2, h \geq 3 .\end{cases}
$$


Since

$$
t=\left\lceil\frac{\left(\begin{array}{c}
d+3 \\
3
\end{array}\right)}{d+1}\right\rceil= \begin{cases}\frac{(h+1)(3 h+2)}{2} & \text { for } \quad d=3 h ; \\
\frac{(h+1)(3 h+4)}{2} & \text { for } \quad d=3 h+1 \\
10 & \text { for } \quad d=5 \\
19 & \text { for } \quad d=8 \\
\frac{3(h+1)(h+2)}{2}+1 & \text { for } \quad d=3 h+2, h \geq 3\end{cases}
$$

then, except for $d=5$ and $d=8$, by [13, Theorem 0.1] we immediately get $\operatorname{dim}\left(I_{X}\right)_{d}=0$.

We remain with the cases $d=5 ; s=9$ and $d=8 ; s=18$. We omit the proves of these cases.

(i) (f) immediately follows from [13, Theorem 0.1$]$.

\section{References}

[1] J. Alexander and A. Hirschowitz, Polynomial interpolation in several variables, J. Algebraic Geom. 4 (1995), 201-222.

[2] M. C. Brambilla and G. Ottaviani, On the Alexander-Hirschowitz theorem, J. Pure Appl. Algebra 212 (2008), 1229-1251.

[3] E. Carlini, M. V. Catalisano and A. V. Geramita, Bipolynomial Hilbert functions, J. Algebra 324 (2010), 758-781.

[4] E. Carlini, M. V. Catalisano and A. V. Geramita, 3-dimensional sundials, Cent. Eur. J. Math. 9 (2011), 949-971.

[5] E. CARlini, M. V. Catalisano and A. V. Geramita, Reduced and non-reduced linear spaces: lines and points, preprint, arXiv:1308.6796

[6] C. Ciliberto, F. Cioffi, R. Miranda and F. Orecchia, Bivariate Hermite interpolation and linear systems of plane curves with base fat points, In: "Computer Mathematics", Lecture Notes Ser. Comput., Vol. 10, World Sci. Publ., River Edge, NJ, 2003, 87-102.

[7] M. V. Catalisano, A. V. Geramita and A. Gimigliano, Higher secant varieties of Segre-Veronese varieties, In: "Projective Varieties with Unexpected Properties", Walter de Gruyter GmbH \& Co. KG, Berlin, 2005, 81-107.

[8] K. A. CHANDLER, A brief proof of a maximal rank theorem for generic double points in projective space, Trans. Amer. Math. Soc. 353 (2001), 1907-1920.

[9] M. Dumnicki, Symbolic powers of ideals of generic points in $\mathbb{P}^{3}$, J. Pure Appl. Algebra 216 (2012), 1410-1417.

[10] M. Dumnicki, B. Harbourne, T. Szemberg and H. Tutaj-Gasiska, Linear subspaces, symbolic powers and Nagata type conjectures, Adv. Math 252 (2014), 471-491.

[11] A. V. Geramita, Inverse systems of fat points: Waring's problem, secant varieties of Veronese varieties and parameter spaces for Gorenstein ideals, In: "The Curves Seminar at Queen's”, Vol. X (Kingston, ON, 1995), Queen's Papers in Pure and Appl. Math., Vol. 102, Queen's Univ., Kingston, ON, 1996, 2-114. 
[12] E. Guardo, B. Harbourne and A. VAn TuYl, Asymptotic resurgences for ideals of positive dimensional subschemes of projective space, Adv. Math. 246 (2013), 114-127.

[13] R. HARTSHORNE and A. HiRsChOWITZ, Droites en position générale dans l'espace projectif, In: “Algebraic Geometry (La Rábida, 1981)", Lecture Notes in Math., Vol. 961, Springer, Berlin, 1982, 169-188.

[14] B. HARbOURne and J. RoÉ, Linear systems with multiple base points in $\mathbb{P} 2$, Adv. Geom. 4 (2004), 41-59.

[15] A. IARrobino And V. Kanev, "Power Sums, Gorenstein Algebras, and Determinantal Loci”, Lecture Notes in Mathematics, Vol. 1721, Springer-Verlag, Berlin, 1999.

[16] A. LAFACE and L. Ugaglia, On a class of special linear systems of $\mathbb{P} 3$, Trans. Amer. Math. Soc. 358 (2006), 5485-5500.

[17] R. MiRAnDA, Linear systems of plane curves, Notices Amer. Math. Soc. 46 (1999), 192201.

[18] S. YANG, Linear systems in $\mathbb{P} 2$ with base points of bounded multiplicity, J. Algebraic Geom. 16 (2007), 19-38.

Dipartimento di Scienze Matematiche

Politecnico di Torino

Corso Duca degli Abbruzzi, 24

10129 Torino, Italia

enrico.carlini@polito.it

DIME

Dipartimento di Ingegneria Meccanica Energetica, Gestionale e dei Trasporti

Università degli Studi di Genova

Piazzale Kennedy, pad. D

16129 Genova, Italia

catalisano@dime.unige.it

Department of Mathematics and Statistics

Queen's University

Kingston, Ontario, Canada, K7L 3N6

and

Dipartimento di Matematica

Università di Genova

Genova, Italia

geramita@dima.unige.it 